\title{
Piecewise Analytic Method VS Runge-Kutta Method (Comparative Study)
}

\author{
Tamer Ahmed Abassy ${ }^{1 *}$ \\ ${ }^{1}$ Department of Scientific Computing, Faculty of Computers and Artificial intelligence, Benha University, Egypt. \\ *Corresponding authorE-mail: Tamerabassy@yahoo.com; Tamerabassy@fci.bu.edu.eg
}

\begin{abstract}
Even though Runge-Kutta (RK) method is the most used by scientists and engineers, it is not the most powerful method. In this paper, a comparative study between Piecewise Analytic Method (PAM) and RK methods is achieved. The result of comparative study shows that PAM is more powerful and gives results better than RK Methods. PAM can be considered as a new step in the evolution of solving nonlinear differential equations.
\end{abstract}

Keywords: Nonlinear Differential Equation; Padé Approximants; Piecewise Analytic Method; Runge-Kutta Method.

\section{Introduction}

Engineers and scientists most important problem is obtaining an accurate solutions to differential equation (DE). The study and application of ordinary DE has been a major part of the history of mathematics. In recent years, new applications in such areas as molecular mechanics and nanophysics have simply added to its significance [1]. The need for analytic methods of high accuracy is very important to understand the mechanism and physical effects through the model problem. For example, problems in the field of astronomy usually have to be solved with high accuracy demands [2].

In numerical analysis, the RK method (RK)is an important method for obtaining the approximate solutions of ordinary DE. It is one of the most used method by scientists and engineers. The $4^{\text {th }}$ order formula is known as the RK formula. It is named for its creators Carl Rung(1856-1927) [3], and Wilhelm Kutta (1867-1944) [4], which the idea was used for $1^{s t}$ order equations. It has been used to obtain approximate numerical solutions of DE. It transforms second and higher orders into a system of equations of first order.

The piecewise analytic method (PAM) was introduced by Abassy for solving any linear or nonlinear initial value ordinary DE [5]-[9]. It is a middle method between numerical and analytic methods and solves critical problems in both. In PAM, the order of accuracy can be controlled according to our needs.

This paper compares the RK method which is the most famous, popular and well known method for solving DE and the new method which is called piecewise analytic method (PAM).

\section{Runge-Kutta Method}

Consider the general 1st order DE:

$$
u^{\prime}=f(t, u), \quad u\left(t_{0}\right)=c .
$$

Runge-Kutta method depends on the idea of using a weighted average of the slopes at $v$ points very close to the current point i.e. it considers the following difference approximation of (1) at the grid point $t_{i}$ with $v$ slopes :

$$
\begin{gathered}
U_{i+1}=U_{i}+\sum_{r=1}^{v} w_{r} K_{r}, \\
K_{r}=h f\left(t_{i}+\varepsilon_{r} h, U_{i}+\sum_{j=1}^{r-1} a_{r j} K_{j}\right), \quad \varepsilon_{1}=0, \quad r=1,2, \ldots, v .
\end{gathered}
$$


where $\varepsilon_{2}, \varepsilon_{3}, \ldots, \varepsilon_{v}, a_{2 j}, \ldots, a_{v(v-1)}$ are parameters to be determined. Since the choice of $\varepsilon_{2}, \varepsilon_{3}, \ldots, \varepsilon_{v}, a_{2 j}, \ldots, a_{v(v-1)}$ in (2) is in no way unique, one can construct a variety of RK formulas which have the same order. The popular RK formulas for various orders are:

A second-order formula [3]

$$
\begin{aligned}
U_{i+1} & =U_{i}+\frac{1}{2}\left(K_{1}+K_{2}\right), \\
K_{1} & =h f\left(t_{i}, U_{i}\right), \\
K_{2} & =h f\left(t_{i}+h, U_{i}+K_{1}\right) .
\end{aligned}
$$

A $3^{r d}$ order formula of Heun [10]

$$
\begin{aligned}
U_{(i+1)} & =U_{i}+\frac{1}{4}\left(K_{1}+3 K_{3}\right), \\
K_{1} & =h f\left(t_{i}, U_{i}\right), \\
K_{2} & =h f\left(t_{i}+\frac{h}{3}, U_{i}+\frac{K_{1}}{3}\right), \\
K_{3} & =h f\left(t_{i}+\frac{2 h}{3}, U_{i}+\frac{2 K_{2}}{3}\right) .
\end{aligned}
$$

By far the most popular formula is the Kutta $4^{\text {th }}$ order formula. It is often referred to as "RK4", "classical Runge-Kutta method" or simply as "the Runge-Kutta method"[4]

$$
\begin{aligned}
& U_{i+1}=U_{i}+\frac{1}{6}\left(K_{1}+2 K_{2}+2 K_{3}+K_{4}\right), \\
& K_{1}=h f\left(t_{i}, U_{i}\right), \\
& K_{2}=h f\left(t_{i}+\frac{h}{2}, U_{i}+\frac{K_{1}}{2}\right), \\
& K_{3}=h f\left(t_{i}+\frac{h}{2}, U_{i}+\frac{K_{2}}{2}\right), \\
& K_{4}=h f\left(t_{i}+h, U_{i}+K_{3}\right) .
\end{aligned}
$$

A relatively popular $5^{\text {th }}$ order formula is that of Nystrom $[11,12,13]$

$$
\begin{aligned}
& U_{i+1}=U_{i}+\frac{1}{192}\left(23 K_{1}+125 K_{3}-81 K_{5}+125 K_{6}\right) \\
& K_{1}=h f\left(t_{i}, U_{i}\right), \\
& K_{2}=h f\left(t_{i}+\frac{h}{3}, U_{i}+\frac{K_{1}}{3}\right), \\
& K_{3}=h f\left(t_{i}+\frac{2 h}{5}, U_{i}+\frac{1}{25}\left(4 K_{1}+6 K_{2}\right)\right), \\
& K_{4}=h f\left(t_{i}+h, U_{i}+\frac{1}{4}\left(K_{1}-12 K_{2}+15 K_{3}\right)\right), \\
& K_{5}=h f\left(t_{i}+\frac{2 h}{3}, U_{i}+\frac{1}{81}\left(6 K_{1}+90 K_{2}-50 K_{3}+8 K_{4}\right)\right), \\
& K_{6}=h f\left(t_{i}+\frac{4 h}{5}, U_{i}+\frac{1}{75}\left(6 K_{1}+36 K_{2}+10 K_{3}+8 K_{4}\right)\right) .
\end{aligned}
$$

For other Runge-Kutta formulas of $5^{\text {th }}$ order are given by Sarafyan $[14,15]$. The $6^{\text {th }}$ order formula of Butcher [16]

$$
\begin{aligned}
& U_{i+1}=U_{i}+\left(\frac{11 K_{1}}{120}+\frac{27 K_{3}}{40}+\frac{27 K_{4}}{40}-\frac{4 K_{5}}{15}-\frac{4 K_{6}}{15}+\frac{11 K_{7}}{120}\right), \\
& K_{1}=h f\left(t_{i}, U_{i}\right) \\
& K_{2}=h f\left(t_{i}+\frac{h}{3}, U_{i}+\frac{K_{1}}{3}\right) \\
& K_{3}=h f\left(t_{i}+\frac{2 h}{3}, U_{i}+\frac{2 K_{2}}{3}\right) \\
& K_{4}=h f\left(t_{i}+\frac{h}{3}, U_{i}+\frac{K_{1}}{12}+\frac{K_{2}}{3}-\frac{K_{3}}{12}\right), \\
& K_{5}=h f\left(t_{i}+\frac{h}{2}, U_{i}-\frac{K_{1}}{16}+\frac{9 K_{2}}{8}-\frac{3 K_{3}}{16}-\frac{3 K_{4}}{8}\right) \\
& K_{6}=h f\left(t_{i}+\frac{h}{2}, U_{i}+\frac{9 K_{2}}{8}-\frac{3 K_{3}}{8}-\frac{3 K_{4}}{4}+\frac{K_{5}}{2}\right) \\
& K_{7}=h f\left(t_{i}+h, U_{i}+\frac{9 K_{1}}{44}-\frac{9 K_{2}}{11}+\frac{63 K_{3}}{44}+\frac{18 K_{4}}{11}-\frac{16 K_{6}}{11}\right) .
\end{aligned}
$$


Table 1

\begin{tabular}{|l|l|l|l|l|}
\hline $\begin{array}{l}\text { The order of the for- } \\
\text { mula } m\end{array}$ & $\begin{array}{l}\text { The number of new } \\
\text { equations of order } m\end{array}$ & $\begin{array}{l}\text { The total number of } \\
\text { equations for order } m\end{array}$ & $\begin{array}{l}\text { Number of stages } n \\
\text { knowns } \frac{n(n+1)}{2}\end{array}$ & \begin{tabular}{l} 
The number of un- \\
\hline 1
\end{tabular} \\
\hline 2 & 1 & 1 & 1 & 3 \\
\hline 3 & 2 & 2 & 2 & 6 \\
\hline 4 & 2 & 4 & 3 & 10 \\
\hline 5 & 4 & 8 & 4 & 21 \\
\hline 6 & 9 & 17 & 6 & 28 \\
\hline 7 & 20 & 37 & 7 & 45 \\
\hline 8 & 48 & 85 & 9 & 66 \\
\hline 9 & 115 & 200 & 11 & 15 \\
\hline 10 & 286 & 486 & 17 & 120 \\
\hline
\end{tabular}

A $7^{\text {th }}$ order formula is introduced by Sarafyan [17] and An eighth-order formula is introduced by Shanks [18, 19]. Formulas of order greater than 8 with exact coefficients are not readily available. These are given more conveniently with approximate coefficients $[18,20]$. Other convenient formulas available in $[1,20,21,22,23]$.

The disadvantages of the RK methods are that they involve considerably more computation per step. For example, RK $4^{\text {th }}$ order formula needs 4 calculations of $f(x, y)$. On the other hand, known formulas of the $5^{\text {th }}$ order, such as the Kutta-Nystrom formula, need 6 calculations of $f(x, y)[11,13]$. The $6^{\text {th }}$ order formulas of A. Huta [24, 25] have the disadvantage of needing 8 calculations of $f(x, y)$. Concentrating on the class of explicit RK methods[26, 27, 28], the difficulties in constructing such a method grow exponentially with the order. This is because the number of nonlinear algebraic equations to satisfy the order conditions is a rapidly increasing function of the order and, to solve this system is far from trivial. In table 1, an idea of the dimension of this system is given. For higher orders it is still an open question, how many stages are at least required? For example, starting with an s-stage explicit RK method providing $\frac{s(s+1)}{2}$ free parameters [2]. Hairer [20] succeeded in satisfying the 1205 conditions for order 10 using only 17 stages, i.e., with 153 parameters[20]. Within the class of explicit RK methods, this is the highest order obtained.

\section{Piecwise Analytic Method}

Consider the $n^{\text {th }}$ order DE

$$
\begin{gathered}
\frac{d^{n} u(t)}{d t^{n}}=f\left(t, u, u^{\prime}, \ldots, u^{(n-1)}\right), \\
u\left(t_{0}\right)=c_{0}, \quad u^{\prime}\left(t_{0}\right)=c_{1}, \quad \ldots, \quad u^{(n-1)}\left(t_{0}\right)=c_{(n-1)} .
\end{gathered}
$$

For solving (8) using PAM, figure 1 explains briefly and diagrammatically the main steps in PAM.

The first step, a general approximate analytical solution for each subinterval which is named by $U_{m}(t)$ is obtained through rewriting equation (8) in the form

$$
\begin{gathered}
\frac{d^{n} U_{m}(t)}{d t^{n}}=f\left(t, U_{m}, U_{m}^{\prime}, \ldots, U_{m}^{(n-1)}\right), \\
U_{m}\left(t_{m}\right)=C_{m, 0}, \frac{d U_{m}}{d t}\left(t_{m}\right)=C_{m, 1}, \ldots, \quad \frac{d^{n-1} U_{m}}{d t^{n-1}}\left(t_{m}\right)=C_{m, n-1}, \quad t \in\left[t_{m}, t_{m+1}\right], \quad m=0,1,2, \ldots .
\end{gathered}
$$

where

$$
U_{m}(t)=\sum_{i=0}^{s} K_{m, i}\left(t-t_{m}\right)^{i}
$$

and $s$ is the order of PAM. Substituting by(10) into (9) and equating the coefficients of $\left(t-t_{m}\right)^{i}$ to zero to get a successive relations that express the coefficient $K_{m, i}$ in terms of the coefficients $K_{m, a}$ where $a<i$. At the end of this step the general approximate analytical solution $U_{m}(t)$ is obtained

$$
U_{m}(t)=g\left(t, t_{m}, K_{m, 0}, K_{m, 1}, \ldots, K_{m, n-1}\right) \quad t \in\left[t_{m}, t_{m+1}\right]
$$

The second step is dividing the solution interval into subintervals, each of length $h$. The subintervals are separated by the nodal points $t_{m}=t_{0}+m h, m=0,1,2, \ldots, n$.

The third(final) step, $U_{m}(t)$ (equation (11)) is applied to each interval successively using $K_{m, 0}=C_{m, 0}=U_{m-1}\left(t_{m}\right)$ where $U_{-1}\left(t_{0}\right)=c_{0}$, $K_{m, 1}=C_{m, 1}=\frac{d U_{m-1}}{d t}\left(t_{m}\right)$ where $\frac{d U_{-1}}{d t}\left(t_{0}\right)=c_{1}, \ldots$ and $K_{m, n-1}=\frac{C_{m, n-1}}{(n-1) !}=\frac{1}{(n-1) !} \frac{d^{n-1} U_{m-1}}{d t^{n-1}}\left(t_{m}\right)$ where $\frac{d^{n-1} U_{-1}}{d t^{n-1}}\left(t_{0}\right)=c_{(n-1)}$.

Note, in some cases (like case 2 in this paper), the results of (10) is not accepted. For solving this problem, the truncated series solution (10) is transformed to Padé approximants

$$
U_{m}(t)=\frac{P_{l}}{Q_{k}}=\frac{\sum_{n=0}^{l} p_{m, n}\left(t-t_{m}\right)^{n}}{\sum_{n=0}^{k} q_{m, n}\left(t-t_{m}\right)^{n}}, \quad l+k=s,
$$

which gives excellent results. For more details see ([5]-[9]). 


\section{Piecewise Analytic Method (PAM)}

2. Dividing the total solution interval into sub-

intervals with length $\boldsymbol{h}$.

Sub-interval $m$ is $t_{m} \leq t \leq t_{m+1}$
1. Finding a general

approximate analytic solution formula $U_{m}(t)$ for

the limited interval $m$ with accuracy $O\left(h^{s}\right)$.

\section{Calculating and applying the obtained solution $U_{m}(t)$ to each} subinterval successively. $(m=0,1,2,3, \ldots)$

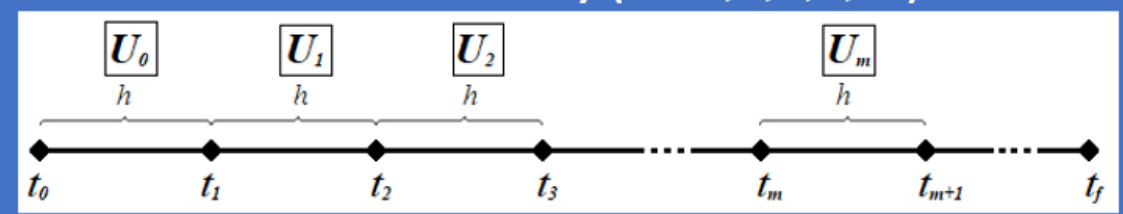

Figure 1: The main steps of PAM.

\section{Case Studies}

\subsection{Case Study 1}

Consider the nonlinear homogeneous DE which has constant coefficients

$$
u^{\prime}-u^{2}+u=0, \quad u(-10)=1 .
$$

It has the exact solution

$$
u(t)=\frac{e^{-t}}{e^{-t}+1}
$$

For solving equation(13) using PAM, rewrite it in the form

$$
\begin{gathered}
U_{m}^{\prime}(t)-U_{m}(t)^{2}+U_{m}(t)=0, \quad U_{m}\left(t_{m}\right)=C_{m, 0}, \\
t \in\left[t_{m}, t_{m+1}\right], \quad m=0,1,2, \ldots, t_{0}=-10, \quad C_{0,0}=1 .
\end{gathered}
$$

substituting by (10) in (15) for calculating the coefficients $K_{m, i}$ setting $s$ according to our needs, for example, if $s=4, K_{m, i}$ 's take the form

$$
\begin{aligned}
K_{m, 0} & =C_{m, 0}, \\
K_{m, 1} & =C_{m, 0}\left(C_{m, 0}-1\right), \\
K_{m, 2} & =\frac{1}{2} C_{m, 0}\left(2 C_{m, 0}^{2}-3 C_{m, 0}+1\right), \\
K_{m, 3} & =\frac{1}{6} C_{m, 0}\left(6 C_{m, 0}^{3}-12 C_{m, 0}^{2}+7 C_{m, 0}-1\right), \\
K_{m, 4} & =\frac{1}{24} C_{m, 0}\left(24 C_{m, 0}^{4}-60 C_{m, 0}^{3}+50 C_{m, 0}^{2}-15 C_{m, 0}+1\right) .
\end{aligned}
$$

If our needs is changed to $s=6$. In this case, the previous results will be taken as it is and add

$$
\begin{aligned}
& K_{m, 5}=\frac{1}{120} C_{m, 0}\left(120 C_{m, 0}^{5}-360 C_{m, 0}^{4}+390 C_{m, 0}^{3}-180 C_{m, 0}^{2}+31 C_{m, 0}-1\right), \\
& K_{m, 6}=\frac{1}{720} C_{m, 0}\left(720 C_{m, 0}^{6}-2520 C_{m, 0}^{5}+3360 C_{m, 0}^{4}-2100 C_{m, 0}^{3}+602 C_{m, 0}^{2}-63 C_{m, 0}+1\right),
\end{aligned}
$$

by the same way

$$
\begin{aligned}
K_{m, 7} & =\frac{1}{5040} C_{m, 0}\left(5040 C_{m, 0}^{7}-20160 C_{m, 0}^{6}+31920 C_{m, 0}^{5}-25200 C_{m, 0}^{4}+10206 C_{m, 0}^{3}-1932 C_{m, 0}^{2}+127 C_{m, 0}-1\right), \\
K_{m, 8} & =\frac{1}{40320} C_{m, 0}\left(40320 C_{m, 0}^{8}-181440 C_{m, 0}^{7}+332640 C_{m, 0}^{6}-317520 C_{m, 0}^{5}+166824 C_{m, 0}^{4}-46620 C_{m, 0}^{3}+6050 C_{m, 0}^{2}-255 C_{m, 0}+1\right) .
\end{aligned}
$$




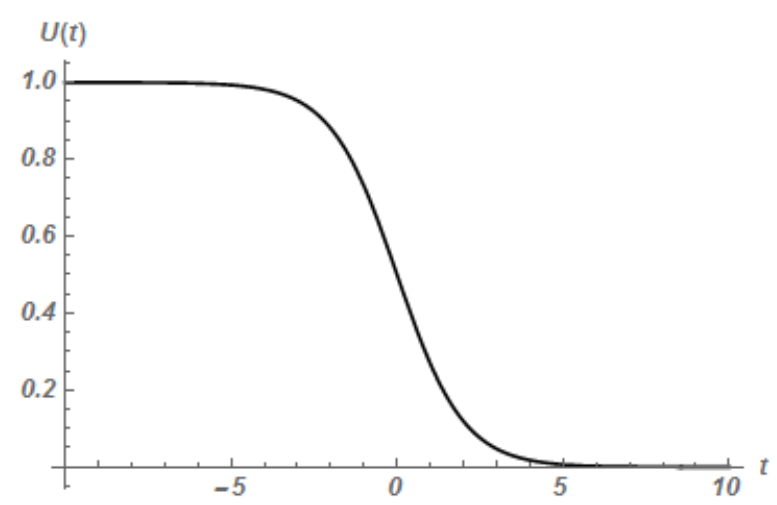

Figure 2: PAM solution for (13) using $h=0.01, t_{0}=-10$ and $s=4$

Then substitute by the obtained $K_{m, i}$ 's in the needed order of the piecewise analytic series solution

$$
U_{m}(t)=\sum_{i=0}^{s} K_{m, i}\left(t-t_{m}\right)^{i}
$$

Figure 2 shows the PAM series solution for (13) using $h=0.01$ and $s=4, t_{0}=-10$ and $C_{m, 0}=U_{m-1}\left(t_{m}\right)$ where $U_{-1}\left(t_{0}\right)=c_{0}=1$.

For solving equation (13) using RK formulas , equation (13) is rewritten in the form

$$
u^{\prime}=f(t, u)=u^{2}-u, \quad u(-10)=1 .
$$

After substitution in RK $K_{i}$ 's in section 2:

The $2^{\text {nd }}$ order RK formula (3) takes the form

$$
\begin{aligned}
& U_{i+1}=U_{i}+\frac{1}{2}\left(K_{1}+K_{2}\right), \\
& K_{1}=h\left(U_{i}^{2}-U_{i}\right) \\
& K_{2}=h\left(\left(U_{i}+K_{1}\right)^{2}-\left(U_{i}+K_{1}\right)\right) .
\end{aligned}
$$

The $3^{\text {rd }}$ order RK formula (4) takes the form

$$
\begin{aligned}
& U_{(i+1)}=U_{i}+\frac{1}{4}\left(K_{1}+3 K_{3}\right), \\
& K_{1}=h\left(U_{i}^{2}-U_{i}\right), \\
& K_{2}=h\left(\left(U_{i}+\frac{K_{1}}{3}\right)^{2}-\left(U_{i}+\frac{K_{1}}{3}\right)\right), \\
& K_{3}=h\left(\left(U_{i}+\frac{2 K_{2}}{3}\right)^{2}-\left(U_{i}+\frac{2 K_{2}}{3}\right)\right),
\end{aligned}
$$

The $4^{\text {th }}$ order formula "The classical Runge-Kutta method" (5) takes the form

$$
\begin{aligned}
& U_{i+1}=U_{i}+\frac{1}{6}\left(K_{1}+2 K_{2}+2 K_{3}+K_{4}\right), \\
& K_{1}=h\left(U_{i}^{2}-U_{i}\right), \\
& K_{2}=h\left(\left(U_{i}+\frac{K_{1}}{2}\right)^{2}-\left(U_{i}+\frac{K_{1}}{2}\right)\right), \\
& K_{3}=h\left(\left(U_{i}+\frac{K_{2}}{2}\right)^{2}-\left(U_{i}+\frac{K_{2}}{2}\right),\right. \\
& K_{4}=h\left(\left(U_{i}+K_{3}\right)^{2}-\left(U_{i}+K_{3}\right)\right) .
\end{aligned}
$$

By the same way calculate the fifth-order formula (6) and the sixth-order formula (7). Tables 2 and 3 show the effect of changing order on the absolute error between the exact solution and each of PAM series solutions and RK solutions for equation (13) using $h=0.1$ and $h=0.01$ respectively.

\subsection{Case Study 2}

Consider the nonlinear non homogeneous DE

$$
u^{\prime}-u^{2}-1=0, \quad u(0)=0 .
$$

It has the exact solution

$$
u(t)=\tan t .
$$


46

International Journal of Applied Mathematical Research

Table 2: The absolute error between the exact solution and each of PAM series solution and RK solution for equation (13) for different orders using $h=0.1$.

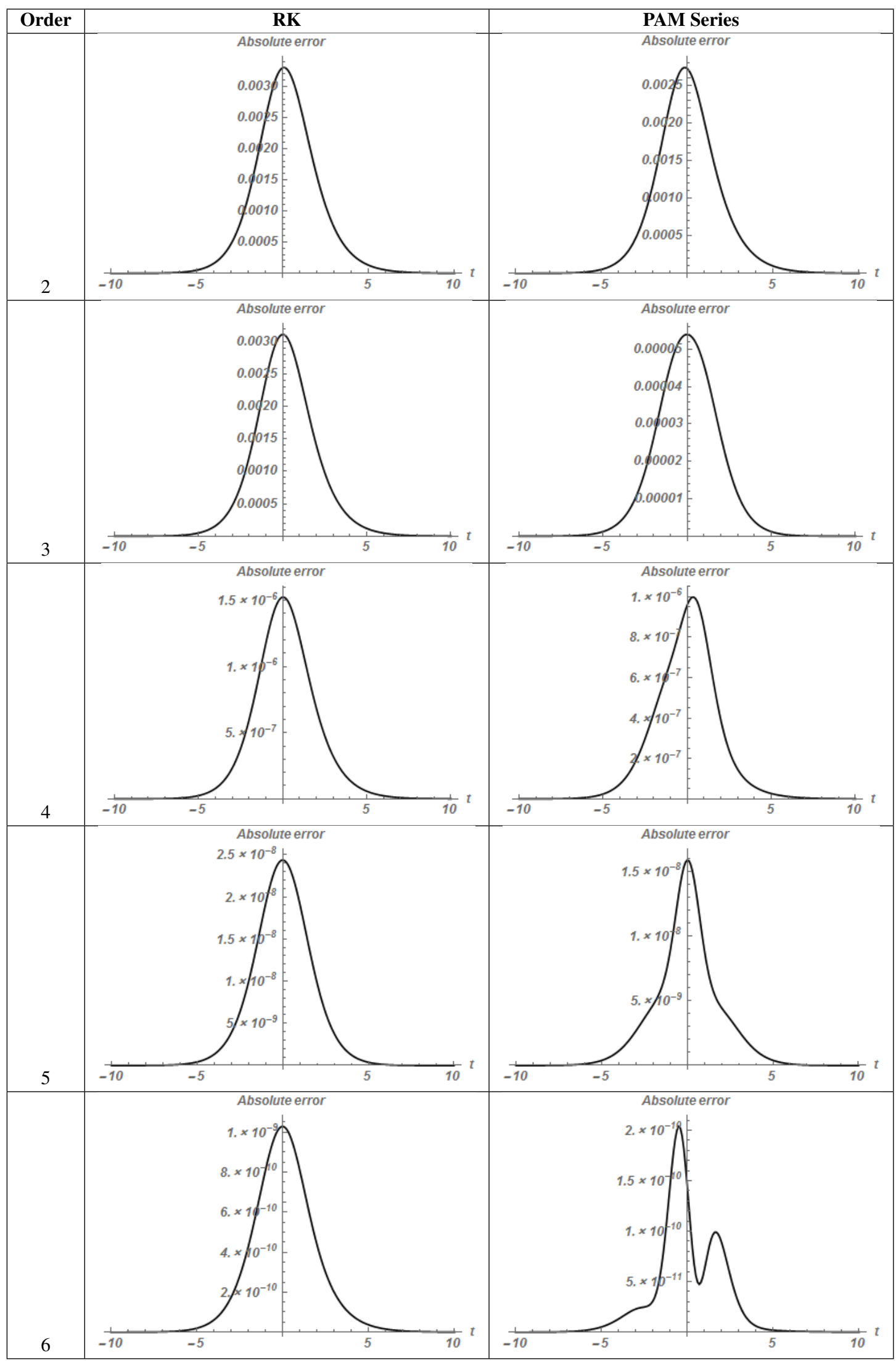


International Journal of Applied Mathematical Research

47

Table 3: The absolute error between the exact solution and each of PAM series solution and RK solution for equation (13) for different orders using $h=0.01$.

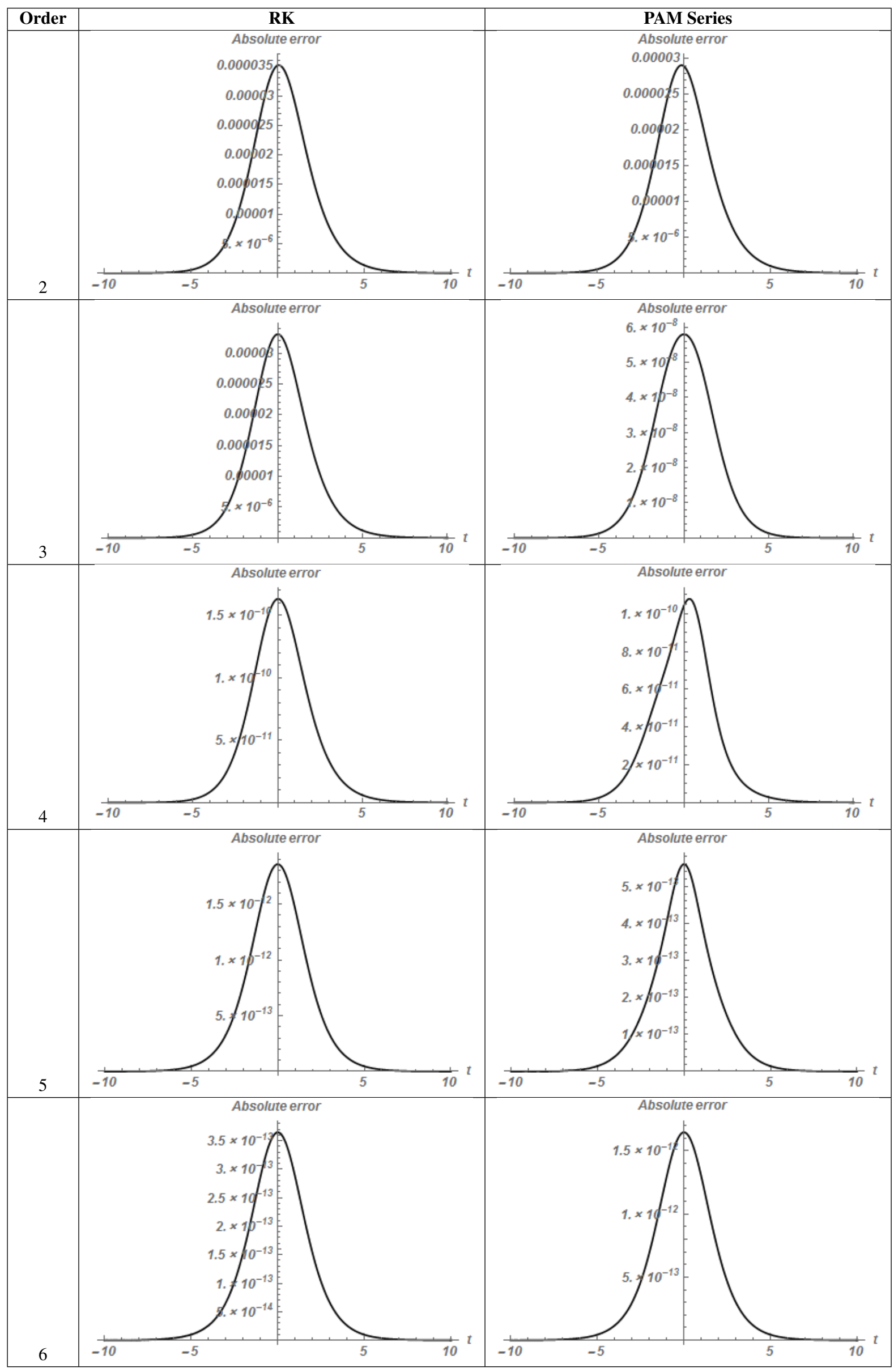




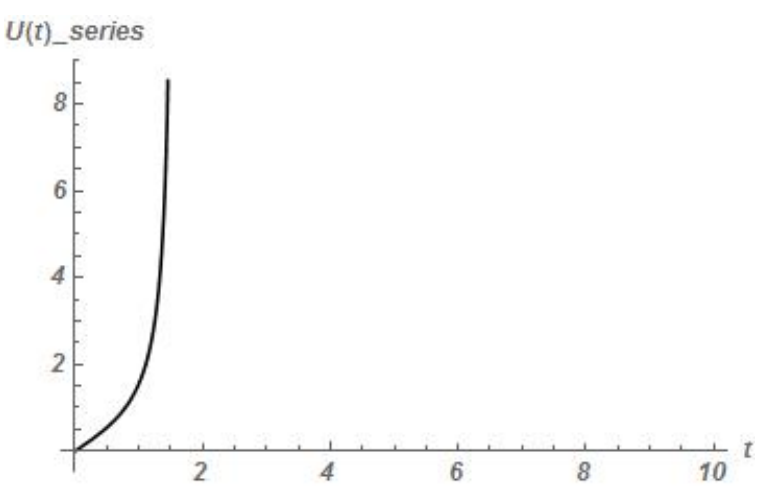

(a) PAM series solution

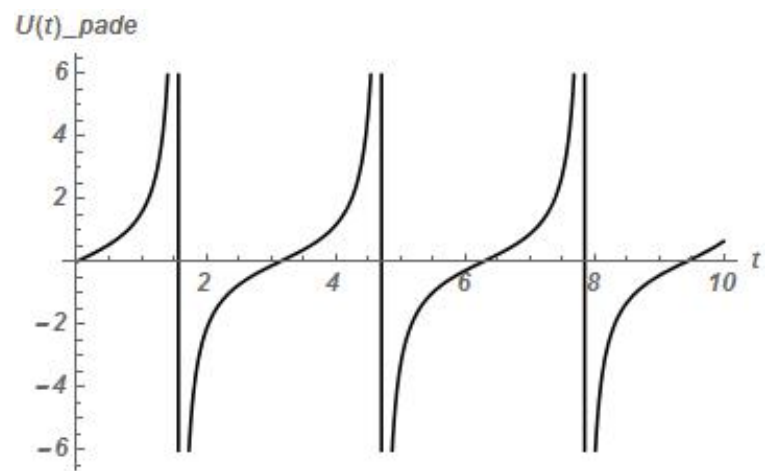

(b) PAM Padé [2/2] solution

Figure 3: PAM solution for (24) using $h=0.01, t_{0}=0, C_{0,0}=0$ and $s=4$

The solution has infinite number of singular points, if equation (24) is solved using any form of RK formulas, it will give me unacceptable solution at all. Also, the piecewise series solution gives me unacceptable solution but the piecewise Padé solution (12) gives me an excellent solution.

For obtaining PAM Padé solution, rewriting equation (24) in the form

$$
\begin{gathered}
U_{m}^{\prime}(t)-U_{m}(t)^{2}-1=0, \\
U_{m}\left(t_{m}\right)=C_{m, 0}, \quad t \in\left[t_{m}, t_{m+1}\right], \quad m=0,1,2, \ldots,(n-1), t_{0}=0, \quad C_{0,0}=0 .
\end{gathered}
$$

substituting by (10) in (26) using $s=4$, the piecewise series solution and $K_{m, i}$ 's take the form

$$
\begin{aligned}
U_{m}(t) & =\sum_{i=0}^{4} K_{m, i}\left(t-t_{m}\right)^{i}, \\
K_{m, 0} & =C_{m, 0}, \\
K_{m, 1} & =\left(C_{m, 0}^{2}+1\right), \\
K_{m, 2} & =C_{m, 0}\left(C_{m, 0}^{2}+1\right), \\
K_{m, 3} & =\frac{1}{3}\left(3 C_{m, 0}^{4}+4 C_{m, 0}^{2}+1\right), \\
K_{m, 4} & =\frac{1}{3} C_{m, 0}\left(3 C_{m, 0}^{4}+5 C_{m, 0}^{2}+2\right) .
\end{aligned}
$$

Using (27) to obtain the Padé approximants $[l / k]=[2 / 2]$.

$$
U_{m_{(\text {pade })}}(t)=\frac{C_{m, 0}\left(t-t_{m}\right)^{2}-3\left(t-t_{m}\right)-3 C_{m, 0}}{\left(t-t_{m}\right)^{2}+3 C_{m, 0}\left(t-t_{m}\right)-3}
$$

Figure 3a shows the PAM series solution (27) for problem (24). From figure, it can be recognized that the PAM series solution (27) is unreliable like RK in this case of study because the solution of (24) is unbounded and has singular points. This problem can be solved by using Padé approximants. Figure $3 \mathrm{~b}$ shows the PAM Padé solution (28) for problem (24). Figure 4 shows the effect of changing $h$ on the absolute error between the exact solution (25) and PAM Padé solution (28) for problem (24).

\section{Comparing PAM with RK Method}

From the previous review and the case of studies, we can summarize the comparison results in the following points:

1. PAM has an Analytic form: In RK, only numerical values can be obtained, but in PAM, in addition to obtaining numerical values, an approximate analytic form can be obtained which can be used in differentiation and integration .

2. PAM order is not limited and easy to increase: The difficulties in constructing higher order forms grow linearly in PAM but exponentially in RK. In RK the number of nonlinear algebraic equations to satisfy the order conditions is a rapidly increasing function of the order and, to solve this system is far from trivial ([26]-[28]).

3. PAM needs less steps and calculations: For example in the studied cases, for calculating $U_{i+1}$ using $U_{i}$ in RK 4 one needs to calculate $4 \mathrm{~K}$ 's first, but in PAM just direct calculation in one step. PAM is not need.

4. PAM formula is unique: The parameters to be determined in RK method Using a Taylor expansion and the order of accuracy are in no way unique, for example, a variety of second-order Runge-Kutta formulas can be construct.

\section{Conclusion}

It can be seen through the obtained results and the comparison between the piecewise analytic method (PAM) and RK method, that PAM is more powerful than RK method. PAM is not a traditional approximate method, it is a moderate method between numerical and analytic method. Strongly nonlinear DE can be solved by PAM. Scientists and engineers can use it for studying and knowing the effects of changing the parameters, coefficients and initial conditions on the solution of DE, in addition to this, PAM controls the accuracy and error very easily according to our needs. 


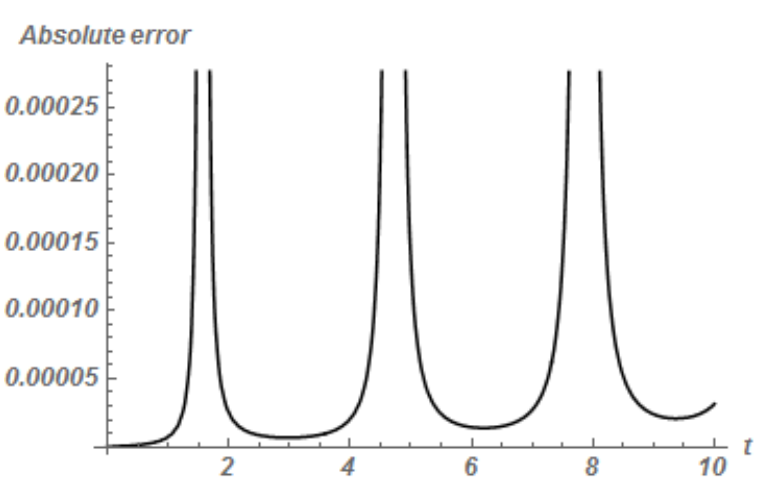

(a) $\left[U_{m}^{(h=0.1)}(t)-\right.$ Exact $]$

Absolute error

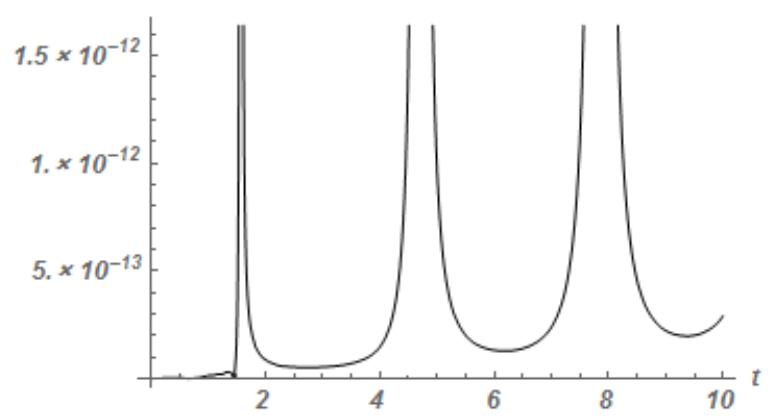

(c) $\left[U_{m}^{(h=0.001)}(t)-\right.$ Exact $]$

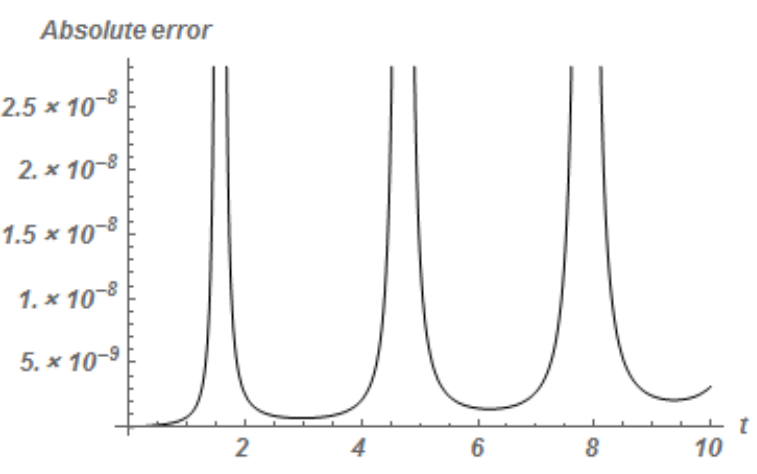

(b) $\left[U_{m}^{(h=0.01)}(t)-\right.$ Exact $]$

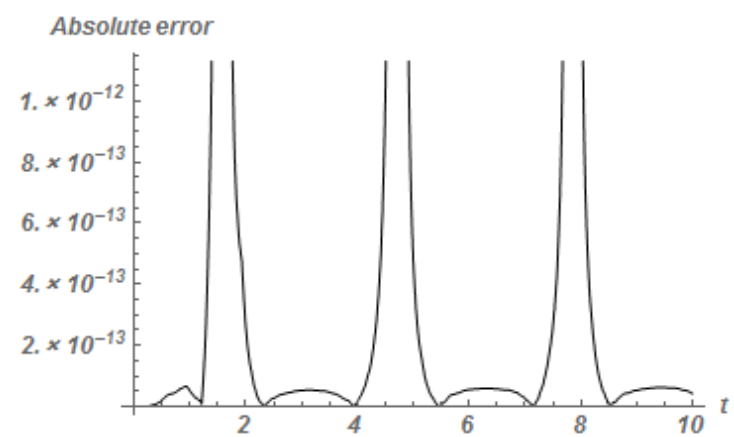

(d) $\left[U_{m}^{(h=0.0001)}(t)-\right.$ Exact $]$

Figure 4: The absolute error between the exact solution (25) and Padé solution (28) for different values of $h$ using $s=4, t_{0}=0$ and $C_{0,0}=0$.

\section{References}

[1] D. Greenspan, Numerical Solution of Ordinary Differential Equations: for Classical, Relativistic and Nano Systems, WILEY-VCH Verlag GmbH and Co. KGaA ISBN: 978-3-527-40610-4, 2006.

[2] B.P. Sommeijer, An Explicit Runge-Kutta Method of Order Twenty-five, CWI Quarterly, 11(1998) p.75-82.

[3] C. Runge, Uber die numerische Auflosung von Differentialgleichungen. Math. Ann. 46(1895) p. 167-178.

[4] W. Kutta, Beitrag zur näherungweisen Integration totaler Differentialgleichungen Z. Math. Phys. 46(1901) p. $435-453$.

[5] T. A. Abassy, Piecewise analytic method, International Journal of Applied Mathematical Research 1(2012) p. 77-107.

[6] T. A. Abassy, Introdction to piecewise analytic method, Journal of Fractional Calculus and Applications 3(S) (2012), 1-19.

[7] T. A. Abassy, Piecewise Analytic Method (Solving Any Nonlinear Ordinary Differential Equation of 1st Order with Any Initial Condition), International Journal of Applied Mathematical Research 2 (2013), no.1, 16-39.

[8] T. A. Abassy, Solving nonlinear 2nd order differential equations using piecewise analytic method (Pendulum Equations), Proceeding of the 13 ${ }^{\text {th }}$ International Conference on Computational Structures Technology, 2018 Sep 4-6; Barcelona, Spain. Elsevier.

[9] T. A. Abassy, Piecewise Analytic Method (PAM) is a New Step in the Evolution of Solving Nonlinear Differential Equations, International Journal of Applied Mathematical Research, 8 (1) (2019) 12-19. doi: 10.14419/ijamr.v8i1. 24984.

[10] K. Heun, Neue Methode zur approximativen Integration der Differentialgleichungen einer unabhängigen Veränderlichen. ZAMP, 45(1900) p. 23.

[11] E.J. Nystrom, Über die numerische Integration von Differentialgleichungen. Acta Soc. Sci. Fenn., 1925. 50(13): p. 1-55.

[12] H. A. Luther, H. P. Konen, Some Fifth-Order Classical Runge-Kutta Formulas. Journal of the Society for Industrial and Applied Mathematics, 4(1965), p. $551-558$

[13] M.K. Jain, Numerical Solution of Differential Equations. 2nd Ed.,Wiley Eastern Ltd. New Delhi, 1984.

[14] D. Sarafyan, Continuous approximate solution of ordinary differential equations and their systems. Comp. Math. Applic. 10( 1984) p. 139.

[15] D. Sarafyan, New algorithms for the continuous approximate solution of ordinary differential equations and the upgrading of the order of the processes. Comp. Math. Applic. 20(1990) p. 77.

[16] J.C. Butcher, On Runge-Kutta processes of high order, J. Aust. Math. Soc. 4(1964) p. 179-194.

[17] D. Sarafyan, 7th-order 10-stage Runge-Kutta formulas. TR 38, Math. Dept., LSU in New Orleans, 1970.

[18] A.R. Curtis, High-order explicit Runge-Kutta formulae, their uses, and limitations. JIMA 16(1975) p. 35.

[19] E.B. Shanks, Solution of differential equations by evaluations of functions. Math. Comp. 20(1966) p. 21.

[20] E. Hairer, A Runge-Kutta method of order 10. J. IMA 21(1978) p. 47

[21] E. Fehlberg, New high order Runge-Kutta formulas with an arbitrarily small truncation error. ZAMM 46(1966) p. 1.

[22] E. Fehlberg, Classical fifth-, sixth- seventhand eighth-order Runge-Kutta formulas with step size control. NASA, TR-R-287, Marshall Space Flight Center, Huntsville, Ala., 1968.

[23] H.A. Luther, An explicit sixth-order Runge-Kutta formula. Math. Comp. 22(1968) p. 344

[24] A. Huta, Une amélioration de la méthode de Runge-Kutta-Nyström pour la résolution numérique des équations différentielles du premier ordre. Acta Fac. Nat. Univ. Comenian. Math. 1(1956) p. 201-224.

[25] A. Huta, Contribution à la formule de sixième ordre dans la méthode de Runge- Kutta-Nyström. Acta Fac. Nat. Univ. Comenian. Math. 2(1957) p. 21-24.

[26] J.C. Butcher, Numerical Analysis of Ordinary Differential Equations: Runge-Kutta and Linear Methods. Wiley, New York, 1987.

[27] J.C. Butcher, Numerical methods for ordinary differential equations in the 20th century. Journal of Computational and Applied Mathematics, 125(2000) p. $1-29$.

[28] J.C. Butcher, Numerical Methods for Ordinary Differential Equations. New York: John Wiley and Sons, 2003. 